\title{
La representación de contextos urbanos en Sendebar. la ciudad y sus peligros
}

\author{
The representation of urban contexts in Sendebar: the city and its dangers
}

\author{
Florencia L. Miranda \\ miranda.florencia@gmail.com \\ Seminario de Edición y Crítica Textual. Instituto \\ de Investigaciones Bibliográficas y Crítica Textual. \\ Universidad de Buenos Aires / CONICET, Argentina
}

\section{Recepción: 30 Octubre 2020 \\ Aprobación: 24 Abril 2021 \\ Publicación: 03 Mayo 2021}

Cita sugerida: Miranda, F. L. (2021). La representación de contextos urbanos en Sendebar: la ciudad y sus peligros. Olivar, 21(33), e097. https://doi.org/10.24215/18524478e097
Resumen: Este artículo se enfocará en un tema que ha sido en general pasado por alto por los estudiosos del Sendebar castellano, que es el de la representación literaria de los contextos urbanos, en especial en su vinculación con los crímenes, delitos, y todo lo que es considerado negativo en la cosmovisión de la literatura ejemplar. En el presente trabajo se abordará el tema desde dos perspectivas complementarias; por un lado, se analizará la forma en que estas representaciones urbanas se articulan con el discurso ejemplar en el que se inserta la colección, y fundamentalmente con los discursos relacionados con los peligros que acechan a la vida humana en comunidad; y, simultáneamente, se tendrá en cuenta la relevancia que tiene el origen oriental del texto y las estrategias de adaptación y asimilación que atraviesan para adaptarse al contexto castellano bajomedieval. Dado que Sendebar se tradujo en la época de emergencia de la prosa ficcional castellana, estas tempranas representaciones de la vida urbana cobran especial relevancia para comprender la forma en que la literatura incipiente representa la cotidianeidad de ciertos ambientes y espacios.

Palabras clave: Sendebar, Literatura Ejemplar, Ciudad, Pecado, Prosa ficcional.

Abstract: The aim of this article is the study of a subject that has generally been overlooked by castillian Sendebar scholars, that is, the literary representation of urban contexts, particularly associated with crime, felonies, among other things that are considered negatively in the exemplary literature's cosmovision. In this article, this subject will be adressed in two complementary perspectives: on the one hand, we will analyse how these urban representations interact with the exemplary discourse of this collection of tales; on the other hand, we will take into consideration the relevance of the oriental origin of these text, and the strategies for cultural adaptation and assimilation that this text had to experience in order to adapt to the Castillian Medieval context. Considering that Sendebar was translated during the period of the emergence of Castilian fictional prose, these early representations of urban life are particularly significant to comprehend the many forms that the medieval literature represents the everyday life of places and environments.

Keywords: Sendebar, Exemplary Literature, City, Sin, Fictional prose. 


\section{INTRODUCCIÓN: EL SENDEBAR HISPÁNICO Y LAS VERSIONES ÁRABES}

Sendebar es una colección de relatos ejemplares traducida del árabe al castellano por mandato del infante Fadrique, hermano menor del rey Alfonso X, en la temprana fecha de 1253, lo que lo convierte en uno de los primeros testimonios de prosa ficcional en castellano, junto con obras como Calila e Dimna. ${ }^{1}$ Este carácter fundacional de la colección, que surge en la Península Ibérica en el contexto de emergencia de la prosa ficcional castellana, le otorga al texto una relevancia que no ha podido ser opacada siquiera por la multiplicidad de errores y lagunas del único manuscrito conservado, que en ocasiones ha complicado tanto su edición como el análisis posterior.

El hecho de que se trate de un texto traducido directamente del árabe que tiene una frondosa familia textual que lo precede y sucede ${ }^{2}$ vuelve necesario situar al Sendebar castellano en su contexto de traducción pero sin olvidar el fenómeno de apropiación y traducción cultural que conlleva su pasaje de un entorno árabe e islámico ${ }^{3}$ a un contexto castellano y cristiano. Es con este objetivo en mente que considero que el análisis de la colección hispánica debe ser llevado a cabo con una puesta en vinculación simultánea, cuando se considere necesario, con versiones árabes conservadas. Si bien estas no pueden concebirse de ninguna manera como fuentes directas de la traducción, conservan rasgos que las acercan al texto castellano y pueden servir como elementos de cotejo para profundizar la comprensión del texto hispánico así como del fenómeno de la traducción y adaptación de la obra.

Si bien las versiones árabes conservadas de Sindibad ${ }^{4}$ no son unidades ficcionales autónomas sino que forman parte de colecciones más amplias que las engloban, el parecido tanto en el relato marco como en los apólogos insertados es evidente. Las mil y una noches contiene una versión de este ciclo de relatos que se conoce tanto con el nombre de "El relato del príncipe y los siete visires" como "La malicia de las mujeres". Con estos dos títulos podemos encontrarlo en las sucesivas ediciones de Las mil y una noches que tienen como fuente el impreso conocido como Calcutta II (1839-1842) que está compuesto de cuatro volúmenes y se considera que está basado en una serie de manuscritos egipcios (Irwin, 2009, 44). Si bien se trata de una versión muy posterior a la que presenta el texto hispánico, ${ }^{5}$ tanto la estructura del relato marco como una gran cantidad de apólogos son coincidentes con el Sendebar castellano (Lacarra, 2009, pp. 69-70). ${ }^{6}$ En los últimos años, la crítica especializada en el Sendebar ha comenzado a prestarle atención a otro ciclo de relatos llamado Las cien y una noches, ${ }^{7}$ que constituye un modelo independiente de Las mil y una noches y probablemente anterior y más arcaico- ${ }^{8}$ y que, se supone, es la versión árabe conservada más cercana al arquetipo de la colección hispánica. Con este panorama en mente, se procederá a examinar algunos apólogos de la colección castellana, refiriéndonos a las versiones árabes cuando el análisis lo requiera.

\section{ConteXtos urbanos EN EL SENDEBaR}

El Sendebar hispánico ha sido objeto de la crítica a lo largo del siglo XX y principios del XXI, en particular por el tono misógino de sus relatos; la declaración que se encuentra en el prólogo, donde se afirma que el libro se escribió "para apercebir a los engañados e los asayamientos de las mugeres" (2019, p. 50) ${ }^{9}$ contribuyó con la fama del libro como una diatriba antifemenina y no permitió profundizar otros aspectos de la colección. El carácter defectuoso de la única copia conservada también dificultó el análisis de esta obra, 
dificultad que hoy en día puede zanjarse, en mayor o menor medida, con la consulta a otras versiones de la rama oriental de Sindibad, como las dos árabes mencionadas previamente.

En el afán por iluminar aspectos del texto que han sido ignorados con anterioridad, propongo partir de la definición de Paul Zumthor de la literatura como la proyección imaginaria de un espacio social (1993, p. 364) y situar el enfoque en las numerosas representaciones de contextos urbanos que forman parte de diversos apólogos, con el objetivo de profundizar el registro de situaciones de la vida cotidiana que se produce en textos fundacionales de la prosa castellana como el Sendebar.

Si bien el relato marco está situado en un ambiente cortesano, contiene una variedad de apólogos que ubican su acción en contextos urbanos y citadinos y la forma en que estos son representados puede servirnos para comprender la forma en que la literatura incipiente representa la cotidianeidad de ciertos ambientes y espacios, representación particularmente relevante por el carácter temprano de estas narraciones en el contexto ficcional castellano. Se destaca en una primera lectura el vínculo entre la cotidianeidad urbana plasmada en los relatos y situaciones de criminalidad y peligrosidad que se entroncan con el discurso ejemplar de la colección, que puede ser considerada como un espejo de príncipes, que tiene como objetivo la educación de los miembros más encumbrados de la corte. Es así que, si bien una importante porción de los peligros que esta colección pone en primer lugar con el objetivo de aleccionar a los cortesanos se vincula de manera directa con el ambiente de la corte, existe un segundo entorno, que es el de la vida en la comunidad urbana, que también despliega una serie de trampas y engaños que el hombre sabio debe conocer de antemano para evitar ser presa de los mismos. Con el objetivo de explorar los vínculos entre el contexto urbano y los peligros y trampas de los que el discurso ejemplar alerta a sus receptores, se analizarán los relatos Panes, Pallium y Senex caecus, ${ }^{10}$ que ocupan los lugares cuarto, décimo tercero y vigésimo segundo en la colección.

\section{PANES: LOS PELIGROS DE COMER EN LA CIUDAD}

Este relato, narrado por el segundo privado con el objetivo de disuadir al rey de la conducta precipitada, ${ }^{11}$ introduce la figura de un mercader, que era "muy señérigo e apartado en su comer e en su bever" (p. 73). El énfasis en la moderación del hombre, que se repite en las versiones árabes, funciona como un agravante de la revelación final y puede considerarse un mecanismo anticipatorio que refuerza la atención en los detalles que es característica de algunos apólogos de esta colección. ${ }^{12}$ La acción se sitúa en "una cibdat muy buena", en la que el mercader se encuentra de paso, y envía a su mozo al mercado para que le consiga comida. En este punto, a pesar de tratarse de un relato breve cuya descripción del ambiente es prácticamente nula, puede destacarse la imbricación entre la ambientación de la ciudad medieval y la centralidad del mercado como lugar de intercambio de bienes. ${ }^{13} \mathrm{~A}$ la vez, en este relato, la ambientación urbana funciona como un punto en un itinerario de un personaje que se encuentra en movimiento: la ciudad constituye un refugio temporal que responde al dinamismo del mercader y se manifiesta como la contrapartida del carácter estático de los personajes de la corte que forman parte del relato marco.

Luego de varios días consumiendo "panes de adárgama" (p. 73), que el mozo conseguía en el mercado, sobreviene la revelación de que el ingrediente central de los panes era una suerte de cataplasma que la cocinera le hacía a su padre, para curarle unas ampollas, con harina de adárgama, manteca y miel. El descubrimiento de los verdaderos ingredientes del pan lleva al mercader a dar "grandes bozes del gran asco que avía" (p. 74) y finaliza con una reconvención a su mozo: “iMezquino! ¿Qué faré que busquemos con que lavemos nuestras manos e nuestros pies e nuestras bocas e nuestros cuerpos? ¿Cómo los lavaremos?” (p. 74). ${ }^{14}$

Sobre este relato ha dicho Pedrosa Bartolomé "Que éste es uno de los cuentos más enigmáticos de todo el Sendebar lo prueba el hecho de que, hasta hoy, los críticos no hayan logrado averiguar nada más sustancial sobre él que su dependencia de Las mil y una noches" (2003, p. 103). La falta de bibliografía abundante sobre este relato puede explicarse también por su brevedad, a la vez que no se encuadra en ninguna tradición 
narrativa específica (como, por ejemplo, lo que sucede en el caso de los relatos sobre adulterio y misoginia). El Index de Stith Thompson lo ubica en el N 383.2 "Man falls dead when he realizes that he has eaten bread from flour used for abscess plaster” $(1958,2018)$.

Eloísa Palafox, en un estudio sobre el rol de la comida en Sendebar, afirma que, en Panes, el elemento central es el efecto del asco, como consecuencia de la ingestión de un producto contaminado: "El asco surge [...] de la idea, a menudo infundada, de que la ingestión o el contacto con un objeto contaminado provoca un cambio dañino e irreversible que puede afectar no solo al cuerpo sino también al alma" (2008, p. 70). La lectura que hace Palafox sobre la ejemplaridad de este relato equipara a los panes contaminados con las mentiras de la madrastra: "el asco y el deseo de evitar comerse el pan contaminado harán que el rey sienta un rechazo instintivo a 'tragarse' la calumnia de la madrastra y matar al infante" (2008, p. 70).

La visión de Palafox me parece sumamente acertada, y solo pondré el foco en el detalle de que el mercader envía a un sirviente para que le consiga alimento, lo que replica de alguna manera una situación de verticalidad similar a la que se presenta en el relato marco entre el rey y el resto de la corte, que incluye a sus privados y a su mujer. El mercader, lógicamente, delega en su sirviente la tarea de conseguir alimento, y su error consiste en no interiorizarse de manera consciente en el tipo de alimento que le es provisto. De esta forma, puede afirmarse que el mensaje subyacente a este apólogo también está relacionado con la excesiva confianza que una figura de autoridad delega en su entorno y en la peligrosidad que se esconde en situaciones cotidianas como, en este caso, la ingesta de alimento. La vinculación especular con la situación planteada en el relato marco es directa: si seguimos la lectura de Palafox, si el mercader representa al rey, y los panes envenenados representan la mentira de la mujer, el énfasis está puesto principalmente en el desconocimiento de la verdadera naturaleza de las cosas, un mensaje que calza perfectamente con la situación del rey Alcos. Por otra parte, el golpe de efecto que conlleva el descubrimiento final del relato funciona como un llamado de atención tanto para el rey, que es el receptor ficcional, como para los receptores extratextuales: la sensación de asco que se produce al imaginar al personaje ingiriendo la cataplasma opera incluso en un lector contemporáneo.

Las versiones de Las mil y una noches y Las cien y una noches son muy similares, y solo contienen algunas alteraciones mínimas que no afectan a la historia de fondo; en algunas versiones, el hombre no se sana, sino que muere; la mujer que hace los panes a veces es la hija del enfermo y a veces es una enfermera. El efecto del asco es igual en todas las versiones, así como el planteo de la imposibilidad de "lavarse" el desagrado, que se ha traducido en la colección castellana de manera poco clara.

El trasfondo urbano de este relato enfatiza la existencia de peligros y potenciales situaciones desagradables vinculadas con el desconocimiento de ciertos sectores y procesos de producción que implica la vida en la ciudad: el hecho de que el mozo no supiera la procedencia del pan es posible gracias al nivel de anonimato característico de un conglomerado urbano, por un lado, y al carácter errante de la figura del mercader, que se encuentra de paso en una ciudad de la que, tanto él como su mozo, desconocen sectores y elementos fundamentales. La conjugación de estos dos factores -el carácter dinámico de un personaje que llega a una ciudad que alberga potenciales peligros virtualmente desconocidos para él- es un motivo reiterado en la literatura medieval en general y en las colecciones de relatos ejemplares en particular. ${ }^{15}$ El dinamismo y la circulación, que hilvanan secuencias narrativas, a la vez pueden funcionar como una metáfora del accionar humano, que debe resguardarse de la vida en comunidad que alberga peligros.

Si bien, por lo que sabemos hasta el momento, este relato tiene un origen árabe, ${ }^{16}$ la adaptación en el contexto castellano se hace de manera prácticamente aproblemática -con excepción de la frase final del mercader-. Esto se relaciona con un aspecto clave del Sindibad como colección, que es la naturaleza secular de sus narraciones y contextos, que permiten una adaptación sencilla y sin necesidad de alterar conceptos fundamentales del contenido del texto. Asimismo, la carencia de descripciones del ambiente -característica de los apólogos breves- permite situar la acción en entornos culturalmente diferentes sin necesidad de modificaciones. En el mismo sentido, el "asco" que sobreviene al descubrimiento de la verdadera naturaleza de los panes está relacionado tanto con la corporalidad -ingerir un alimento vinculado con alguna parte 
del cuerpo humano- como con una enfermedad -ya que se trataba de un compuesto que se utilizaba como un remedio- que constituyen elementos tabú para la ingesta que son comunes a muchas comunidades, lo que implica que el cuento se haya transmitido de manera casi inalterada y sea fácilmente entendible en sus múltiples contextos de traducción. ${ }^{17}$

Así como Paul Zumthor afirmaba, de la ciudad medieval, que rechaza "le nomadisme de la société médiévale : à un univers ambient parcellisé, elle oppose sa massivité ; à l'émiettement des solitudes rurales, à l'isolement des ligngages féodaux, la concentration des êtres et de leurs ressources" (1993, p. 128), esta concentración conlleva una profundización del anonimato y el desconocimiento de ciertas características del entorno que implica, ciertamente, un nivel de peligro. En este caso, no se trata de un engaño deliberado con el objetivo de perjudicar al otro, como en el caso de las representaciones de la corte; ni se trata de un engaño motivado por la debilidad moral, como en el caso de las mujeres adúlteras; simplemente es una suerte de malentendido u omisión que surge como consecuencia de las disposiciones de la convivencia y la distribución de tareas en el contexto urbano bajomedieval.

\section{PALLIUM: EL COMERCIO COMO EXCUSA PARA EL ENGAÑO}

Pallium o el "Enxemplo de la muger e del alcaueta [e] del omne e del mercador que vendió el paño" es el segundo relato del quinto privado y es narrado con el objetivo de descalificar al género femenino para, de esa manera, deslegitimar a la figura de la mujer del rey. Pertenece al motivo K.1543 "The Marked Coat in the Wife's Room" (1958, p. 409) y se encuentra en todas las versiones de la rama oriental del Sendebar, excepto en el Tütì-Nâmeh persa, aunque es una historia que se popularizó también en el ámbito europeo. Sus semejanzas con el $f$ abliau francés Auberée han sido analizadas por Álvaro Galmés de Fuentes, quien, en lo tocante a la relación entre ambos relatos, afirma que "los detalles coincidentes [...] son tantos que no cabe pensar que ambos relatos hayan surgido con independencia en Oriente y Occidente" (1989, p. 6).

La ambientación urbana de este relato se ve enfatizada por su vínculo con la literatura de fabliaux, que se caracteriza tanto por la ubicación de los relatos en un contexto urbano como por la profunda interrelación entre el contenido narrado y la ambientación citadina:

La ciudad define una forma de ser del mundo, a la que corresponden nuevas funciones narrativas como las que aporta el fabliau. Se instaura un espacio de socialización en la medida en que la gran plaza y los mercados invitan a un incesante codearse con el otro, a la familiaridad y a la burla de las que nos da cuenta el ambiente recreado en los textos (Calvo y Chicote, 1997, p. 11).

Este apólogo pertenece a la serie de relatos de tono misógino de la colección, que se avienen a la declaración del prólogo y son narrados con el objetivo de descalificar a la mujer del rey; de los veintitrés relatos que componen el texto, ocho pueden sindicarse como pertenecientes a este conjunto. ${ }^{18} \mathrm{El}$ relato pone en primer lugar la principal acusación que se les imputa a las mujeres en esta colección, que es la de su naturaleza engañadora; el privado introduce el apólogo ante el rey con la frase "los engaños de las mugeres non an cabo nin fin" (p. 100). El argumento del relato, resumido brevemente, narra la historia de una alcahueta que ayuda a un hombre que quiere concretar su deseo por una mujer casada; como esta es una "buena muger" (p. 101) y no atiende a sus requerimientos, la anciana orquesta una trampa que implica la compra de un paño que vende el marido de la mujer, que genera una serie de equívocos que conducen a la mujer, engañada, a un encuentro con el hombre, que consuma su deseo; y, posteriormente, el mismo paño funciona como excusa para que el mercader crea en la inocencia de su esposa y la historia termine sin consecuencias.

La particularidad que tiene este enxemplo es la de poner en primer lugar a dos personajes femeninos, de las cuales una -la alcahueta- es quien orquesta el engaño, para cumplir con el deseo de un hombre; y la esposa, que es quien comete el adulterio, es una víctima pasiva tanto de los recursos engañadores de la vieja como del 
deseo masculino. Esta polaridad entre los dos personajes femeninos de la historia es común a los dos relatos de Sendebar que son protagonizados por alcahuetas y deja en claro el rol corruptor de la anciana mediadora.

Para comprender en su totalidad la naturaleza de este relato es menester detenernos brevemente en la figura de la alcahueta. Sendebar contiene dos de los retratos más tempranos de las alcahuetas o medianeras en la literatura hispánica; una figura que se consagrará en este ámbito siglos después, con personajes como Trotaconventos en el Libro de Buen Amor o la misma Celestina de Fernando de Rojas, da sus primeros pasos en el contexto de la prosa ficcional castellana en los relatos Canicula y Pallium. Si bien hay antecedentes literarios de la figura de la mediadora en la literatura clásica, ${ }^{19}$ el origen oriental de Sendebar vincula directamente a estas ancianas con el modelo árabe de la alcahueta o mediadora, una figura literaria común tanto a la literatura culta árabe como a los relatos populares. ${ }^{20}$ Los rasgos que caracterizan a la alcahueta, en estos relatos, son su ancianidad y su conocimiento de recursos y dispositivos para llevar a cabo distintos engaños, cuyo objetivo último es la facilitación de encuentros adúlteros por los que cobra una compensación económica.

A la vez, la figura de la alcahueta tal como se plantea en este relato se encuentra intrínsecamente imbricada con el contexto urbano. La separación de los habitantes por género, que confina a las mujeres al interior, hace necesaria la aparición de la medianera para llevar a cabo empresas moralmente condenables como el adulterio. De esta forma, la ubicación en un contexto urbano cobra relevancia en la libre circulación de la alcahueta que, por su condición de anciana, cuenta con una mayor impunidad para moverse tanto en ambientes internos -en la versión árabe, por ejemplo, utiliza como excusa las abluciones religiosas para introducirse en la casa de la mujer- como en la calle. Como era de esperarse, en los ambientes internos se encuentra de manera casi exclusiva con el otro personaje femenino de la historia, mientras que en sus salidas al exterior negocia e intercambia ideas con los personajes masculinos, lo que la pone en una situación de exclusividad: la ancianidad, en este caso, funciona como un nivelador ascendente.

Una de las situaciones más retratadas en los apólogos situados en contextos urbanos es la del comercio: en este relato, el hecho de que el esposo de la mujer sea mercader funciona como un elemento en la trama del engaño urdido por la alcahueta. La compra del paño es un dispositivo que pone en marcha el engaño y le otorga a la mujer casada una suerte de salvaguarda para la circulación, similar a la que goza la anciana. El cambio de manos del paño, que circula por distintos ambientes y lugares con el objeto de encubrir el adulterio, también será experimentado por la mujer casada, que será conducida a diferentes ámbitos para lograr satisfacer el deseo del hombre y lograr así que la alcahueta tenga una compensación monetaria por su accionar.

De esta forma, así como en Panes observábamos que la ciudad albergaba peligros por el nivel de anonimato y desconocimiento de ciertos individuos, en este caso el peligro y las malas acciones están vinculadas con la libre circulación de actores sociales como la alcahueta, y las actividades comerciales pueden servir como excusa para llevar a cabo engaños y concretar deseos ilícitos.

Es importante destacar que en las historias situadas en contextos urbanos cobran relevancia motivaciones y justificaciones vinculadas con el rédito económico, con mayor frecuencia que en los apólogos situados en ambientes cortesanos. La representación de la vida urbana está atravesada por la motivación económica, que origina las situaciones de peligro: la alcahueta es un ejemplo paradigmático de las motivaciones "degeneradas" típicas de los contextos de ciudad.

Al mismo tiempo, puede apreciarse que los engaños que se llevan a cabo en los relatos urbanos son más sofisticados y complejos que los que se representan en ambientes cortesanos, donde el "engaño" se materializa como una falsa acusación o una indicación deliberadamente errónea con el objetivo de perjudicar al otro. En el caso de Pallium -y en el relato que se analizará a continuación- las tramas de los engaños implican un nivel de inventiva y una serie de pasos intermedios que los vuelve operaciones elaboradas. 


\section{SENEX CAECUS: LA CIUDAD DE LOS “OMNES MALOS E DE MALA REPUELTA"}

Este relato, el tercero de cuatro que narra el infante luego de recobrar la voz, es una de las historias más largas y complejas del Sendebar. Figura en todas las versiones orientales, salvo en Las cien y una noches dado que el infante no narra ninguna historia luego de su reaparición-y el Tütì-Nâmeh persa. Ben Edwing Perry (1960) destaca un antecedente grecolatino de este apólogo - La vida de Esopo- como un argumento para apoyar su hipótesis del origen persa de la colección $(1960$, p. 15$) .^{21}$

Arbesú en su edición señala, por un lado, el nivel de elaboración de este relato, y, por el otro, la cantidad de incongruencias que presenta el texto. Estas posibles deturpaciones del texto son visibles desde el título del apólogo, "Enxemplo del mercador del sándalo e del otro mercador", que introduce una pareja de mercaderes que no aparece, ya que el relato solo contiene un personaje que oficia de mercader; a la vez que en la introducción del infante se anuncia la "estoria del viejo" (p. 135) que no está mencionado en el título, y deja afuera a los otros protagonistas del relato, el mercader y la vieja. Con respecto a la introducción de la historia, tanto Senex caecus como el siguiente, Abbas, que son los apólogos que cierran la colección, muestran deturpaciones en la inserción de los apólogos en el relato marco: ambos se abren con la pregunta del rey “¿Cómo fue eso?”, pero este interrogante no se encuentra motivado por ninguna frase previa, como en los apólogos anteriores, en los que siempre figuraba una sentencia que funcionaba como pie para la pregunta del rey y el subsiguiente relato. El hecho de que se trate de los apólogos finales que se encuentran contiguos puede hacernos suponer posibles errores del copista, ya sea para reemplazar una porción de texto perdido o por alguna razón que desconocemos.

El relato es el más largo de toda la colección y cuenta con distintas instancias narrativas: se trata de un encadenamiento de tres engaños que sufre un mercader que llega a un pueblo, y que, con la ayuda voluntaria de una anciana e involuntaria de un anciano ciego, logra revertirlos. ${ }^{22}$ Los tres relatos centrales que narra el infante ${ }^{23}$ tienen como objetivo la representación de personajes sabios, que funcionan, a la vez, como una limitación a la sabiduría del infante ("Yo te diré quién sabe más que yo" [127] es la frase con la que introduce sus relatos), y a la vez, como una confirmación de su sapiencia recientemente adquirida. Estos relatos, además, se caracterizan por postular como máximos representantes de la sabiduría a individuos situados en extremos opuestos de la vida (niños y ancianos), vinculados entre sí por el tópico de la ceguera. ${ }^{24}$

En este relato se introduce la imagen de la ciudad con una elocuente sentencia en boca de la vieja, que funciona como una figura positiva y consejera del mercader protagonista: "los de esta villa son engañadores e malos baratadores, e nunca viene omne estraño que non lo escarnescan” (p. 136). De este modo, la relación entre el asentamiento urbano y el engaño y la trampa es directa y explícita. Los engaños de los hombres están relacionados con el comercio, el juego y los pleitos judiciales: se intenta sacar ventaja del mercader en lo que respecta a su cargamento de sándalo, en un juego de dados y, finalmente, el hombre tuerto amaga con armar un pleito judicial ante el alcalde por el supuesto robo de su ojo. ${ }^{25}$

Gracias a su extensión y complejidad, Senex caecus brinda una representación dinámica y completa de la ciudad y de la vida en la comunidad, vinculada con la movilidad del personaje del mercader, que es el protagonista de la historia. Es así que el relato se inicia en las puertas de la ciudad -o villa, que es el otro término que se utiliza en la narración para designar al conglomerado urbano- que es donde el mercader sufre el primer engaño de parte del hombre que quema el incienso para comprárselo a menor precio. El carácter fronterizo de las puertas de la ciudad hace que se cruce con una "manceba que trayé su ganado de pacer" (p. 135), una figura más vinculada con las grandes extensiones de campo por fuera de las murallas de la ciudad, pero que también se conecta con la serie de trampas citadinas, dado que al alertar a otro mercader del pueblo sobre el cargamento de incienso es quien pone en funcionamiento el primero de los engaños, el de la quema de incienso. 
El segundo engaño tiene lugar en el mercado, que constituye uno de los núcleos del trazado urbano y el lugar de encuentro por excelencia. Este segundo engaño, que se produce en un juego de dados, deja en claro que las trampas que pueden acechar al hombre en la ciudad no están únicamente vinculadas con la actividad comercial.

Por último, el tercer engaño sucede en la calle; el hombre que "non avía sinon un ojo" (p. 137) es un estafador profesional que aprovecha su condición de tuerto para acusar al mercader del robo de su ojo. En este caso, asistimos a un típico timo vinculado con la convivencia urbana, donde la extranjería del mercader le juega en contra y lo convierte en la víctima perfecta para los criminales del pueblo.

Esta comunidad de hombres engañadores y tramposos tiene un líder, que es el anciano ciego, a quien acuden todos los días para confesarle sus fechorías en una suerte de asamblea de ladrones. Este anciano va a funcionar como un segundo consejero -involuntario- del mercader, ya que es quien planteará las respuestas necesarias para esquivar cada una de las trampas a las que fue sometido por los hombres del pueblo. En este relato, el consejo para ayudar al protagonista a solucionar su problema será involuntario; la transmisión de saberes, en este caso, es esquiva e indirecta; el mercader escucha a escondidas los consejos del viejo, que no sabe que está revelando información que perjudicará a sus propios hombres; es el buen criterio del mercader -asistido previamente por la vieja- el que lo ayudará a salir del conflicto. Respecto de esta transmisión de saberes, afirma Salvatore Luongo:

Aquí parece importar poco que este use normalmente el saber de manera negativa, a favor de sus secuaces; lo que cuenta es que su sabiduría, aun sin intención, sea puesta ahora a disposición del protagonista. Lo que marca la diferencia en el Sendebar como en obras similares, es el uso, bueno o malo, que se hace del conocimiento; incluso aquel al que recurre la madrastra es una forma de conocimiento, pero usado para un mal propósito, en apoyo de una mentira. (2017, p. 82)

El nivel de complejidad de las trampas requiere de la creatividad y astucia del viejo para sortearlas; el hecho de que se trate de un viejo ciego que lidera una asamblea de ladrones y timadores, que se une a las figuras de los portadores de sabiduría de los relatos anteriores del infante, dos niños “ciegos e contrechos" (p. 129), da una pauta sobre la transmisión de sabiduría en Sendebar. Si bien el infante es reconocido por toda la corte como su integrante más sabio -y por esta misma razón se vuelve merecedor del trono de su padre- el conocimiento de los límites de su propia sabiduría es, paradójicamente, una parte fundamental de su formación y sapiencia. A la vez, el hecho de que el mayor nivel de sabiduría sea ostentado por niños y un anciano ciegos, a los que se suma el hecho de que el anciano lidera una banda de ladrones deja en claro que la sabiduría en Sendebar es un atributo que puede adquirirse en contextos heterogéneos, a la vez que enaltece la humildad demostrada por el infante, que sabe reconocer la sabiduría incluso en personajes y situaciones no exentas de polémica.

\section{A MANERA DE CONCLUSIÓN}

El carácter fundacional del Sendebar en lo que respecta a la prosa ficcional castellana lo vuelve un objeto de estudio relevante por su valor documental tanto de rasgos lingüísticos -aspecto no contemplado en este artículo- como de situaciones y representaciones vinculadas con la vida cotidiana en el contexto castellano bajomedieval. Es así que en una serie de apólogos de esta colección se plasman algunas de las primeras representaciones de contextos y vivencias urbanas en la prosa ficcional castellana. Si bien la crítica mantiene un consenso sobre la circulación cortesana de Sendebar y el peso fundamental que tiene el espacio cortesano en la colección, tanto como escenario central de la acción como el ámbito de recepción de los contenidos, ${ }^{26}$ el hecho de que algunos apólogos estén situados en contextos que se encuentran por fuera de la corte y que ilustren situaciones relativas a la convivencia en comunidad no debe ser pasado por alto.

Si bien el Sendebar es el producto de una traducción del árabe, esto no le resta relevancia a las representaciones que pueda contener que reflejen el contexto castellano bajomedieval. En este ámbito se llevó a cabo un proceso de adaptación de los contenidos al contexto de traducción que, en líneas generales, 
fue aproblemático, y los casos en los que los contenidos no fueron adaptados exitosamente nos brindan información relevante acerca de la complejidad de las relaciones interculturales.

Respecto de los apólogos que están situados en contextos urbanos, puede enumerarse una serie de características en común. En primer lugar, la existencia de un nivel de "profesionalización" del delito en las ciudades; por un lado, encarnado en figuras como la alcahueta, cuyo oficio se enfoca en la concreción de encuentros adúlteros, con o sin el consentimiento de una de las partes; por otro lado, de manera más explícita, en el relato Senex caecus, la representación de la asamblea de ladrones funciona como la institucionalización del "oficio" criminal; "oficio" que tiene un representante -el ciego- cuya sabiduría está a la altura de los personajes sabios de otros relatos, y cuya sapiencia, si es recibida por la persona correcta, puede aprovecharse de manera positiva.

En segundo lugar, el contexto urbano planteado como la aglomeración de un grupo humano se destaca, en estos apólogos, como la representación de un punto fijo en el itinerario de personajes nómades como los mercaderes de Panes y Senex caecus, para quienes la ciudad es un lugar que provee cobijo y oportunidad de concretar negocios. De esta forma, puede afirmarse que la representación de la ciudad como centro productivo que nuclea bienes y recursos, tal como afirma Zumthor (1993, p. 128) es un concepto central en esta colección. Sin embargo, la productividad de la ciudad tiene como contracara la proliferación de peligros que aguardan, principalmente, a los personajes que son externos a la dinámica de la ciudad y, por esa misma razón, desconocen las trampas y engaños que caracterizan a un sector de la misma. La ciudad es peligrosa, sí; pero es particularmente peligrosa para los forasteros u "omnes estraños" que la habitan de manera temporal. De este modo, se retoma la metáfora del viaje y el movimiento como representación de la vida humana; así como el prólogo de la colección lleva a cabo una metáfora náutica y afirma que, el infante Fadrique, mecenas de la obra, "Tomó una nave endereçada por la mar en tal que non tomó peligro en pasar por la vida perdurable" (pp. 49-50), la imagen del mercader que se desplaza entre ciudades también puede considerarse una condensación de la actividad humana y los peligros que ese desplazamiento conlleva.

Por último, el rasgo final a destacar reside en el nivel de complejidad de las trampas citadinas, que están en consonancia con la mencionada profesionalización de la criminalidad. Este rasgo queda en claro particularmente en Senex caecus, donde las trampas están, de alguna manera, institucionalizadas en la asamblea de ladrones y son supervisadas por la figura del ciego, que constituye un referente de la autoridad criminal; aunque, en la misma línea, también debe destacarse la figura de la alcahueta, que está ligada sustancialmente con la ambientación urbana y que recibe una suerte de paga informal por sus intervenciones, que consisten en planes rebuscados y premeditados con astucia.

\section{REFERENCIAS}

Arbesú, D. (Ed.). (2019). Sendebar. Libro de los engaños e los asayamientos de las mugeres. Delaware: Juan de la Cuesta.

Armijo Canto, C. E. (2019). El saber adquirido a través del secreto en el Sendebar, Las Mil y una noches y Las Ciento y una noches. Memorabilia: Boletin de Literatura Sapiencial, 21, 137-150.

Armistead, S. y Monroe, J. (1989). Celestina's muslim sisters. Celestinesca, 13(2), 3-28.

Artola, G. (1978). The Nature of the Book of Sindibad. In H. Niedzielski, H. Runte, \& W. Hendrickson (Eds.), Studies on the Seven Sages of Rome: And Other Essays in Medieval Literature: Dedicated to the Memory of Jean Misrabi. Honolulu: Educational Research Associates.

Belcher, S. (1987). The Diffusion of the Book of Sindbād. Fabula, 28(1), 34-58.

Burton, R. (Ed. y trad.). (1885). The Book of the Thousand Nights and a Night (Vol. VI). Burton Club.

Calvo, F. y Chicote, G. (1997). Caracterización del espacio urbano en los fabliaux. Medievalia, 26, 9-16.

Chauvin, V. (1904). Bibliographie des ouvrages arabes ou relatifs aux Arabes: Publiés dans l'Europe chrétienne de 1810 à 1885 (Vol. 8). Liège: H. Vaillant-Carmanne. 
Comparetti, D. (1869). Ricerche intorno al Libro di Sindibad. Milano: Giuseppe Bernardoni.

Cosquin, E. G. (1909). Le Prologue-Cadre des Mille et une nuits, les légendes perses et le Livre d'Esther. Revue Biblique(1892-1940), 6(1), 7-49.

Douglas, M. (2007). Pureza y peligro. Un análisis de los conceptos de contaminación y tabú. Buenos Aires: Nueva Visión. Esopo. (2001). Fábulas. Vida de Esopo. (P. Bádenas de la Peña, Ed.). Madrid: Gredos.

Fernández, E. (2007). Medianeras y mediadoras: Mujeres y mediación en las letras hispánicas. Revista Canadiense de Estudios Hispánicos, 32(1), 3-8.

Fradejas Lebrero, J. (Ed.). (1990). Sendebar o Libro de los engaños de las mujeres. Madrid: Castalia.

Fudge, B. (Ed. y trad.). (2016). A Hundred and One Nights. New York: New York University Press.

Galmés de Fuentes, Á. (1989). El "Libro de los engaños" y el fabliau francés de "Auberée”. Estudios Románicos, 4, 431439.

Gómez Redondo, F. (1998). Historia de la prosa medieval castellana. I. La creación del discurso prosístico: El entramado cortesano (Vol. I). Madrid: Cátedra.

Grotzfeld, H. (2004). The Manuscript Tradition of the Arabian Nights. En U. Marzolph, R. van Leeuwen, \& H. Wassouf (Eds.), The Arabian Nights Encyclopedia (pp. 17-21). Santa Barbara: ABC-CLIO.

Haro Cortés, M. (2015). De "Balneator" del "Sendebar" a "Senescalus" de los "Siete sabios": Del "exemplo" al relato de ficción. Revista de Poética Medieval, 29, 145-175.

Haro Cortés, M. (2017). De diablos, diablesas y seres extraordinarios en el Sendebar: Los cuentos Striges, Fontes, Simiay Nomina. En A. González \& L. von der Walde Moheno (Eds.),Perspectivas y proyecciones de la Literatura Medieval (pp. 141-172). México: Universidad Autónoma Metropolitana.

Haro Cortés, M. (2018). Del alfayate al elefante en Elephantinus (cuento 16) del Sendebar. En H. O. Bizzarri (Ed.), Monde animal et végetal dans le récit bref du Moyen Âge (pp. 37-54). Wiesbaden: Reichert Verlag.

Hawkins, J. (2011). Seeing the Light? Blindness and Sanctity in Later Medieval England. Studies in Church History, $47,148-158$.

Irwin, R. (2009). The Arabian Nights. A Companion. New York: Tauris Parke Books.

Lacarra, M. J. (2009). Entre el "Libro de los engaños" y los "Siete visires": Las mil y una caras del "Sendebar" árabe. En A. Chraïbi \& C. Ramírez Gomez (Eds.), Les mille et une nuits et le récit oriental: En Espagne et en occident (pp. 51-74). Paris: Harmattan.

Lemos Horta, P. (2015). Beautiful Men and Deceitful Women: The One Hundred and One Nights and World Literature. Narrative Culture, 2(2), 190-207.

Luongo, S. (2017). «Señor, dizen de la estoria del viejo»: El cuento Senex caecus del Sendebar. Memorabilia: Boletín de Literatura Sapiencial, 19, 79-89.

Lyons, M., Lyons, U. e Irwin, R. (Eds. y trads). (2010). The Arabian Nights: Tales of 1,001 Nights (Vol. 1-3). Penguin Classics.

Márquez Villanueva, F. (1991). La Celestina como antropología hispano-semítica. Sharq Al-Andalus, 8, 269-292.

Márquez Villanueva, F. (1993). Origenes y sociología del tema celestinesco. Barcelona: Anthropos.

Marzolph, U., Van Leeuwen, R. y Wassouf, H. (2004). The Arabian Nights Encyclopedia. Santa Barbara: ABC-CLIO.

Marzolph, U. y Chraibi, A. (2012). The Hundred and One Nights: A Recently Discovered Old Manuscript. Zeitschrift Der Deutschen Morgenländischen Gesellschaft, 162(2), 299-316.

Pajón Mecloy, E. (2013). El ciego como figura literaria. Madrid: Antígona.

Palafox, E. (2008). 'E yo tomava aquella masa en escuso e fazíala pan': El lugar de la comida en el exemplum medieval, el caso del Sendebar castellano. Memorabilia: Boletin de Literatura Sapiencial, 11, 65-80.

Pedrosa Bartolomé, J. M. (2003). Pan de adárgama y vino de sorgo: Las mil y una noches (noche 580), el Sendebar (cuento 4), Sorgo rojo de Mo Yan y una vieja historia de Miguel Delibes. Revista de Poética Medieval, 10, 101108.

Perry, B. E. (1960). The Origin of the Book of Sindbad. Fabula, 3(1), 1-94. 
Ramos, R. (2005). Texto, compilador y códice: El relato final del Libro de los engaños. In B. Taylor \& G. West (Eds.), Historicist Essays on Hispano-Medieval Narrative in Memory of Roger M. Walker (pp. 386-407). London: Modern Humanities Research Association.

Robinson, C. y Rouhi, L. (Eds.). (2007). Representing Others in Medieval Iberian Literature (1st edition). New York: Palgrave Macmillan.

Rouhi, L. (1999). Mediation and Love. A Study of the Medieval Go-Between in Key Romance and Near-Eastern Texts. Leiden - Boston - Köln: Brill.

Rozin, P. (1994). Food Enculturation. En B. G. Galef Jr., M. Mainardi, \& P. Valsecchi (Eds.), Behavioral Aspects of Feeding, Basic and Applied Research in Mammals (pp. 203-228). Chur - Langhorne: Harwood Academic Publishers.

Sarmiento, M. (1775). Memorias para la historia de la poesía y poetas españoles. Dadas a luz por el monasterio de $S$. Martin de Madrid y dedicadas al Excmo. Sr. Duque de Medina-Sidonia. Madrid: Joachim Ibarra.

Scarborough, C. (2018). Viewing Disability in Medieval Spanish Texts. Disgraced or Graced. Amsterdam: Amsterdam University Press.

Thompson, S. (1958). Motifindex of folk-literature: A classification of narrative elements in folktales, ballads, mybts, fables, mediaeval romances, exempla, fabliaux, jest-books, and local legends. Bloomington: Indiana University Press.

Turón, M. (1986). El viaje constante de la narrativa medieval. RILCE. Revista de Filología Hispánica, 2(2), 299-314.

Wheatley, E. (2013). Stumbling Blocks Before the Blind. Medieval Constructions of a Disability. Michigan: University of Michigan Press.

Zumthor, P. (1993). La mesure du monde. Paris: Seuil.

\section{Notas}

1 Aunque es menester recordar que la fecha de traducción de Calila e Dimna, si bien se ha aceptado convencionalmente el año 1251, continúa siendo materia de debate; no ocurre lo mismo con el Sendebar, que tiene los datos concretos de su traducción especificados en el prólogo (cfr. Sarmiento, 1775, 339-342, donde se expone por primera vez la hipótesis sobre la fecha de traducción del Calila).

2 La versión hispánica pertenece a la conocida como rama oriental del Sindibad, que cuenta con ocho versiones: siríaca, griega, hebrea, tres versiones persas, dos versiones árabes (conocidas como "El relato del príncipe y los siete visires" o "La malicia de las mujeres", se trata de una colección de relatos que forma parte de Las mil y una noches y de Las cien y una noches) y la traducción castellana. Sobre las distintas ramas del Sindibad, cfr. Comparetti (1869), Chauvin (1904), Perry (1959), Artola (1978) y Belcher (1987).

3 Lamentablemente, la no conservación del original árabe a partir del cual se realizó la traducción al castellano hace que el contexto del que proviene el Sendebar tenga carácter hipotético, aunque es posible conjeturar vínculos del texto hispánico con algunas versiones árabes conservadas, como veremos a continuación.

4 Con el vocablo Sindibad se designará de manera colectiva a los textos pertenecientes a la rama oriental, mientras que Sendebar se aplicará solo al texto castellano, para evitar ambigüedades.

5 Del Sendebar sabemos, por un lado, que su traducción se llevó a cabo a mediados del siglo XIII, mientras que el único manuscrito que lo contiene fue copiado en el siglo XV; respecto del impreso Calcutta II, que es el que contiene el ciclo del "Príncipe y los siete visires", sabemos que está basado en manuscritos egipcios no identificados por el momento; sin embargo, el manuscrito más antiguo en el que figura este ciclo se remonta al siglo XVI (Grotzfeld, 2004, 19). En las ediciones tanto de Burton (1885-1888) como de Lyons (2010) que están basadas en el impreso Calcutta II se evidencia una cantidad de detalles y ampliaciones que pueden considerarse evidencia de una etapa de composición muy posterior a la del texto de la colección hispánica.

6 De hecho hay más coincidencias en los apólogos entre el Sendebar y El ciclo "El príncipe y los siete visires" contenido en Las mil y una noches que entre la rama occidental y la rama oriental del Sindibad.

7 La primera en llamar la atención con contundencia sobre la cercanía entre el Sendebar castellano y el relato del "Príncipe y los siete visires" de Las cien y una noches fue María Jesús Lacarra (2009), seguida por Marta Haro Cortés (2015a; 2015b; 2018) y Carmen Armijo Canto (2019). 
8 A contrapelo de lo que podría parecer en una primera lectura rápida, se trata de una versión que tiene antecedentes más antiguos que Las cien y una noches, su "hermano mayor" literario: la historia proyectada en el relato marco es de origen chino y su primera documentación puede datarse en el año 251 A.D. (Cosquin, 1909, 14), lo que sitúa la composición de Las cien y una noches en una época anterior al de Las mil y una noches, y, también, la postula como una colección menos "occidentalizada" y retocada por manos de los sucesivos editores, dos características que también se explican por la escasa difusión de la colección en Europa. Las referencias más tempranas sobre la existencia de Las cien y una noches se remontan al siglo X (Marzolph et al., 2004, t. II, 595). aunque a juzgar por la antigüedad de la historia narrada en el relato marco, se presume que su existencia podría ser anterior. Respecto del soporte editorial, se conservan doce manuscritos conocidos, aunque muy pocos están datados (Marzolph y Chräibi, 2012; Lemos Horta, 2015, 96). El más antiguo datado es un manuscrito ubicado en la Biblioteca Nacional de París, con la nomenclatura BNF 3662, cuya fecha de composición se sitúa en 1190 A.H./1776 A.D.

9 Las citas del Sendebar castellano, salvo indicación explícita en contrario, se harán tomando como base la edición de David Arbesú (2019), por lo cual a continuación solo se consignará el número de página. Esta elección se funda en el trabajo comparativo que lleva a cabo el editor con otras versiones de la rama oriental (siria, griega, hebrea, persas, árabes) para aclarar pasajes oscuros que considero que sitúa al Sendebar en un contexto multicultural que es necesario para esta colección.

10 Utilizo los nombres latinos de los cuentos tal como se ha popularizado en las ediciones del Sendebar. Respecto de su origen, cfr. Arbesú $(2019,65)$.

11 Sendebar está estructurado en una suerte de contienda entre dos bandos que aconsejan con objetivos contrapuestos: por un lado están los siete privados reales, que quieren evitar que el rey actúe de manera precipitada y mate a su hijo por consejo de su mujer, para lo cual se valen de dos tipos de relatos; los primeros tienen como objetivo desalentar las acciones impulsivas con exempla que ilustran situaciones desgraciadas que les ocurren a los protagonistas que actúan sin premeditación; el segundo relato tiene como objetivo descalificar la actuación femenina en general, para desacreditar de esa forma a la mujer del rey; en el caso de esta, cuenta un solo relato por vez que tiene como objetivo principal injuriar a los privados.

12 Refuerzo esta característica de la composición concluyente y atenta a los detalles de ciertos apólogos para contrapesar las dificultades de lectura que ha presentado a lo largo del tiempo el Sendebar por la defectuosidad de su único manuscrito conservado.

13 No sorprende que Las mil y una noches, que muestra un texto cuya composición es posterior, narre la situación de otra manera: en esta versión, es el mercader quien se encuentra por la calle con una anciana que lleva unos panes que llaman su atención y regatea el precio hasta quedárselos. En este caso, tanto el carácter fortuito del encuentro como la insistencia del hombre en la compra de los panes pueden leerse como agregados posteriores que amplifican el relato y refuerzan la sorpresa de la revelación final.

14 La incongruencia de esta declaración - no queda claro qué es lo que no puede lavarse- se subsana al recurrir a la versión de Las cien y una nohes, donde el mercader hace una declaración final: اغسل فمي وبني فكيف اغسل جوفي (ägsal famī wabuni fakaîfa àgsal yaüfì) que puede traducirse como "Puedo limpiar mi boca y mi cuerpo, pero ¿cómo limpiaré mi interior?" (Fudge [ed.], 2016, 282).

15 Reproduzco las siguientes palabras de Mercedes Turón, que considero pertinentes por su contundencia y poder de síntesis: "No habrá libros de viajes durante el medioevo, pero no hay narración sin viajes" (1986, 312). El viaje como motivo estructurador es una constante de la literatura medieval, así como, en las colecciones ejemplares como Sendebar y Calilae Dimna es un recurso narrativo común la inserción de viajes en unidades narrativas menores como los apólogos, que no modifican la estructura básica del relato marco pero otorgan un contexto para la acción del relato.

16 Este relato se encuentra en todas las versiones orientales con excepción de las persas (Döhla, 2019, 74) lo que nos hace suponer una procedencia árabe; sin embargo, por el momento, no contamos con información adicional que esclarezca de manera definitiva su origen.

17 Respecto del tema específico del asco que suscita la ingesta de alimentos contaminados con residuos patológicos, cfr., además del citado texto de Palafox, Rozin (1994) y Douglas (2007).

18 Se trata de los relatos Avis, Gladius, Canicula, Pallium, Elephantinus, Nomina, Ingenia y Abbas. Los primeros siete son narrados por los privados y el último, que cierra la colección, está en boca del infante; sin embargo, existen hipótesis, bastante firmes, acerca del carácter agregado de este apólogo, por su incongruencia a nivel temático y estilístico (cfr. Ramos, 2005).

19 Respecto de la mediadora o alcahueta como tópico literario en el contexto hispánico, cfr. Armistead y Monroe (1989); Márquez Villanueva (1991; 1993); Rouhi (1999) que en su capítulo 1 hace un repaso por la figura de la mediadora en la Antigüedad Clásica, desde Plauto hasta las Metamorfosis de Ovidio; también Fernández (2007) y Robinson y Rouhi (2007).

20 Acerca de la interacción entre la literatura árabe culta y la popular, y la inserción de la figura de la alcahueta en una intersección de ambas, afirma Rouhi: "From the eighth to the fifteenth centuries, artists produced a court culture, a 
learned culture, and a popular culture, all of which interacted with and informe done another in significant ways. [...] Collections of tales, for example, display the intermingling of popular and learned in their subject matter, and variations on the same basic story can be found both in folkloric and learned sources. By extension, the literary manifestation of the Near-Eastern go-between occurs in both popular and learned literature" (1999, 160).

21 El fragmento narrativo que puede ubicarse en La vida de Esopo es el relativo a la segunda prueba, que implica beber toda el agua del océano, y es el siguiente:

“[...] Dijo Esopo:

-Cuando venga el árbitro de la apuesta con el adversario diciendo que te bebas el mar, no digas que no, al contrario, ponte como si estuvieras borracho. Coge una mesa, ordena que la sirvan y que se presenten los esclavos. Esto dará lugar a un espectáculo; acudirá todo el mudo a verlo porque tú te vas a beber el mar. Cuando veas que está todo lleno de gente, llenas una taza con agua de mar y cuando hayas hecho venir al juez de la apuesta, dí ‘ ¿qué apuesta hice?' y él te dirá 'que te bebas todo el mar'. Y tú dices: ‘'nada más?', 'no', te dirá. Entonces, tú pones testigos de esto y dices: ‘Ciudadanos, muchos son los ríos, hinchados por las lluvias e inagotables, que fluyen al mar. La única apuesta que hice fue beber el mar y no los ríos que desembocan en él. Así que, el adversario cierre las desembocaduras de los ríos, para beber únicamente el mar. Si imposible del todo es cerrar las bocas de los ríos que hay en el mundo, también es imposible que yo me beba el mar'. De este modo, unido un imposible con otro imposible, se romperá la apuesta." (2001, 190-193).

22 Fradejas Lebrero, en su edición, postula que el relato está estructurado en tres partes (engaño, asamblea y juicio) y que todo en el relato gira en torno al número tres: los engaños, los consejos, las sentencias. (1990, 145-146).

23 Los denomino "centrales" porque se encuentran en el nudo de la secuencia narrativa del infante: el que abre la serie es Lac Venenatum, que se relata con el objetivo de espejar la situación vivida previamente en el relato marco y contestar a la pregunta de Alcos acerca de la culpabilidad que le corresponde a cada actor de la historia; y el relato final, $A b b a s$, solo retoma el tópico misógino para enfatizar la necesidad del castigo de la madrastra. Los tres relatos que se narran en el segmento central del discurso del infante tienen como objetivo la confirmación de su sabiduría, que lo hace merecedor del trono de Alcos.

24 Respecto del ciego como tópico literario medieval, cfr. Hawkins (2011), Pajón Mecloy (2013), Wheatley (2013) y Scarborough (2018).

25 La versión de Las mil y una noches presenta un cuarto engaño, que lleva a cabo un zapatero que le arregla una sandalia al mercader e intenta hacerle trampa con el pago; por un lado, puede pensarse que se quiebra la armonía estructural tripartita (cfr. nota xxii), pero, por el otro, se profundiza la multiplicidad de espacios y niveles del engaño citadino.

26 En palabras de Fernando Gómez Redondo: "Varias son las líneas temáticas que atraviesan la obra (la valoración del saber, los engaños de la mujer, las obligaciones de los privados, la educación principesca), pero sólo una da sentido a todas: el análisis del espacio cortesano como asiento de la conducta del Rey" (1998, 218). 